\title{
Incidence of psoriasis in patients with active systemic lupus erythematosus
}

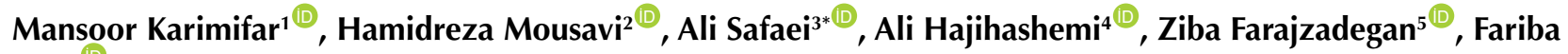 \\ Iraji
}

${ }^{1}$ Departments of Rheumatology, Alzahra Hospital, Isfahan University of Medical Sciences, Isfahan, Iran

${ }^{2}$ Department of Rheumatology, Noor and Aliasghar Hospital, Isfahan University of Medical Sciences, Isfahan, Iran

${ }^{3}$ Isfahan Cardiovascular Research Center, Cardiovascular Research Institute, Isfahan University of Medical Sciences, Isfahan, Iran

${ }^{4}$ Department of Radiology Alzahra Hospital, Isfahan University of Medical Sciences, Isfahan, Iran

${ }^{5}$ Departments of Community Medicine, Isfahan University of Medical Sciences, Isfahan, Iran

${ }^{6}$ Department of Dermatology, AL-Zahra Hospital, Isfahan University of Medical Sciences, Isfahan, Iran

Correspondence to:

Ali Safaei, Email:

alisafaei461993@yahoo.com

Received: 11 July 2020

Accepted: 24 Aug. 2020

ePublished: 18 Sep. 2020

Keywords: Systemic lupus erythematosus, psoriasis, Systemic lupus erythematosus disease activity index

\begin{abstract}
Introduction: Concomitant onset of active systemic lupus erythematosus (SLE) and psoriasis is rare; however, due to the common skin involvement in both and exacerbation of psoriasis with antimalarial medications used for SLE, it is crucial to investigate the concomitant occurrence of both diseases.

Objectives: The present study aims to investigate the incidence of psoriasis in people with active SLE. Patients and Methods: The present cross-sectional study was performed on 148 patients with active SLE from 2015 to 2017. Lupus activity was evaluated based on systemic lupus erythematosus disease activity index$2 \mathrm{~K}$ (SLEDAI-2K), and then, the incidence of psoriasis in patients was investigated. Patients' demographic information was recorded in the relevant checklist.

Results: The mean score of SLEDAI-2K was $14.10 \pm 19.77$. The duration of SLE was significantly associated with the SLEDAI-2K score $(P=0.001, r=0.294)$. Psoriasis incidence was remarkably correlated with the gender of patients with SLE $(P=0.019)$. An insignificant association was found between the coincidences of SLE with psoriasis considering the factors, including age, SLE duration, and mean SLEDAI-2K score.

Conclusion: Patients' mean age, SLE duration, and disease activity score were not correlated with concomitant psoriasis among SLE patients; however, we found that males were more prone to concomitant SLE and psoriasis.
\end{abstract}

\section{Introduction}

Citation: Karimifar M, Mousavi H, Safaei A, Hajihashemi A, Farajzadegan Z, Iraji F. Incidence of psoriasis in patients with active systemic lupus erythematosus. J Prev Epidemiol. 2020;5(1):e07. doi: $10.34172 /$ jpe. 2020.07
The systemic lupus erythematosus (SLE) is an autoimmune disease with unknown etiology; however, systemic inflammatory processes are prominent in this disorder to the extent that it involves several organs of the body. In this term, antibodies against the nucleus and cytoplasm of cells have been investigated to play a significantly destructive role in multiple organs such as the kidneys, heart, central nervous system, musculoskeletal system, and skin. SLE is associated with periods of relapses and remissions (1,2). Like most major autoimmune diseases, lupus is more common in young women of childbearing age, but its severity is not necessarily related to gender (3). SLE is associated with several complications, including secondary thrombosis, antiphospholipid syndrome, carditis, pneumonia, hypertension, major adverse cardiovascular events, inflammation,

\begin{abstract}
Key point
In a study on 148 patients with active systemic lupus erythematosus (SLE), we found, the mean age of SLE patients, the duration of lupus, and the mean score of lupus activity were not associated with psoriasis coexistence, while SLE males were significantly more affected by psoriasis concomitantly as compared to females.
\end{abstract}

and infection of the brain; thus, the relapses may cause catastrophic complications (4). Ultraviolet light (sunlight), medications, some chemicals, viruses, hormonal factors, genetics, and infections (5) are among the factors associated with lupus recurrences.

Various studies have shown that 15\%-72\% of patients with SLE develop skin lesions including malar rash, mucous membrane lesions, alopecia, nonspecific rashes, nail infections, pigment changes, swelling of the limbs and dry skin, Raynaud's phenomena, 
chronic discoid lesions and blisters, swelling around the eyes, urticaria, palmar erythema, vasculitis of the skin, chilblain, psoriasis form lesions and livedo reticularis $(6,7)$.

Searching the literature has shown that autoimmune disorders have the potential of overlapping; for instance, psoriasis like lupus is associated with skin and joint involvement that may overlap with SLE. Psoriasis is one of the most common skin diseases worldwide, with an estimated global prevalence of $0.5 \%$ to $4.6 \%$. Recent studies have shown that about $2.6 \%$ of the population in northern Europe and Scandinavia has psoriasis, and it is also the most common autoimmune disorder in the United States, while it is scarce in Japan. Psoriasis is a chronic inflammatory skin disease involving the immune system in which inflamed, thick, gray, scaly, sometimes itchy patches of skin appear, and the lesions may be burning or painful (8-10). Etiologically psoriasis is a multifactorial disease in which hereditary factors have a significant role. Numerous genetic studies have proven the role of heredity, but there are many environmental factors in the development of psoriasis. The most prominent factors associated with psoriasis are infections, physical injuries, medications, mental, hormonal, and metabolic factors. Although the association of psoriasis with the activity of some underlying diseases such as lupus has been investigated, limited numbers of studies have identified significant relationships between the severity of lupus and psoriasis. In addition, the results of these studies are controversial and there is no comprehensive report in this regard $(9,11)$.

\section{Objectives}

The coincidence of rheumatologic diseases can help better investigate the pathogenesis of the diseases and may impact on the therapeutic approach used for the treatments because of different indications and contraindications related to each of the disorders. Due to the inadequate information about the correlation of psoriasis with SLE and its severity, the present study is aimed to investigate this correlation.

\section{Patients and Methods \\ Study design}

The present cross-sectional study was performed on 148 patients with active SLE who referred to Al-Zahra and Noor clinics (affiliated to Isfahan University of Medical Sciences) from January 2015 to March 2017.

The patients with the diagnosis of SLE based on the American College of Rheumatology (ACR) criteria (12), whose disease was in the active course based on the SLEDAI-2K scoring criteria and presented their willingness to participate in the study were included. Additionally, those patients whose SLE was in remission to the SLEDAI-2K scoring criteria and patients who were unable to track psoriasis for any reason were excluded from the study.
The required information was provided for the participants as well as reassurance for the confidentiality of their personal information, and written consent was obtained.

The necessary tests were taken to determine the SLEDAI-2k. The activity of lupus disease was determined by the SLEDAI-2K scoring system by a skilled target rheumatologist.

The active SLE was defined as the least of a four or an eight score out of 105 points in the SLEDAI-2K scoring system (13).

Patients were then referred to a dermatologist to assess the presence of psoriasis clinically, and those with clinical relevance to psoriatic lesions referred for biopsy and pathological confirmation to confirm the diagnosis of psoriasis (14).

\section{Ethical issues}

The research followed the tenets of the Declaration of Helsinki. The Ethics Committee of Isfahan University of Medical Sciences approved this study. The institutional ethical committee at Isfahan University of Medical Sciences approved all study protocols (\#ir.mui.rec.1396.3.180). Accordingly, written informed consent was taken from all participants before any intervention. This study was extracted from the M.D thesis of Ali Safae at this university (Thesis \#396180).

\section{Data analysis}

The collected data were entered into the Statistical Package for Social Sciences (SPSS) version 22. The descriptive data were presented in mean, standard deviation, absolute numbers, and percentages. Chi-square test, $t$ test, Fisher's exact test, analysis of covariance (ANCOVA), and regression were used for analytics. Accordingly, $P$ value of less than 0.05 was considered as a significant level.

\section{Results}

In the present study, 148 patients with active SLE according to SLEDAI-2K criteria, including six men (4.05\%) and 142 women $(95.94 \%)$ were examined. The mean age of the subjects was $37.65 \pm 12.29$ years, and the duration of their diagnosis was $5.42 \pm 3.60$ years.

The mean score of SLEDAI-2K was 19.77 \pm 14.10 . According to the findings of the present study, among the patients examined, 6 (4.1\%) individuals had psoriasis, among which $2(33.33 \%)$ patients were male, and 4 $(66.66 \%)$ ones were female $(P=0.019)$ (Table 1$)$.

The duration of lupus was not associated with psoriasis $(P=0.316)$. Besides, the $t$ test showed that the mean SLEDAI-2K score was not significantly associated with psoriasis $(P=0.295)$ as well as the patients' age $(P=0.08)$.

Further investigations revealed that the longer duration of lupus had a significant direct correlation with the SLEDAI-2K score $(\mathrm{r}=0.294, P=0.001)$. 
Table 1. The association of active lupus with psoriasis in patients studied

\begin{tabular}{|c|c|c|c|}
\hline & \multicolumn{2}{|c|}{ Occurrence of psoriasis } & \multirow{2}{*}{$P$ value } \\
\hline & Yes & No & \\
\hline Gender & & & 0.019 \\
\hline Male & 2 & 4 & \\
\hline Female & 4 & 138 & \\
\hline Duration of lupus (year) & $5.60 \pm 3.68$ & $5.33 \pm 3.58$ & 0.316 \\
\hline Average age (year) & $40.04 \pm 11.50$ & $36.22 \pm 12.58$ & 0.08 \\
\hline Average SLEDAI-2k score & $14.93 \pm 11.98$ & $22.55 \pm 14.57$ & 0.295 \\
\hline
\end{tabular}

\section{Discussion}

Autoimmune diseases can overlap and may concurrently be detected in a patient, depending on the underlying mechanism. The devastating activity of circulating immune complexes seems to play a major role in SLE, whereas no specific etiology for psoriasis has been raised yet. Although most of the studies have identified psoriasis as a result of T-cell activity, some studies have shown the role of antibodies in the pathogenesis of this disorder. In other words, some studies have shown the role of interleukins in psoriasis that stimulate cytotoxic cells (T-helper type-1) and (T-helper type-2), and these cytokines are the common point found in both lupus and Psoriasis (15-17).

It is crucial to investigate the concomitant occurrence of lupus and psoriasis from two perspectives, one in which up to $72 \%$ of lupus patients present various skin manifestations, which are also the chief complaints of psoriatic patients (6). On the other hand, antimalarial drugs, which are the gold standard treatment for SLE can exacerbate or recur skin involvement in psoriasis $(9,11)$. The present study examined the occurrence of psoriasis in patients with active lupus for the first time.

In the previous reports, the gender distribution of lupus was significantly higher among the female population, and even ratios of 9 to 1 have been reported $(3,18)$. In our study, the proportion of female patients with active lupus was 23 times higher than males. This higher ratio may be due to the higher number of women seeking follow-up compared to men, or the period in which the disease is active in the female population. In the present study, 148 people with active disease were evaluated after the elimination of patients with lupus in remission course. Among those surveyed, lupus activity had a significant association with the duration of the disease, and those patients who had SLE for a more extended period had a higher SLEDAI$2 \mathrm{~K}$ score. Among patients with active lupus, $4.1 \%$ had psoriasis concurrently. The findings of our study showed that despite the higher proportion of females with concomitant psoriasis, there was a significant relationship in the distribution of psoriasis in male patients with SLE. Considering that the present study was performed on patients with active lupus, the obtained results can be evaluated due to the activity of lupus or small statistical population. Therefore, more extensive studies are strongly recommended.
Varada and colleagues, as well as Bethelot et al, presented a considerably lower prevalence of SLE and psoriasis coexistence $(10,19)$.

In a study by Zalla et al, $0.69 \%$ of patients with psoriasis referred with the manifestations of lupus, and $1.1 \%$ of the patients with the documented SLE developed psoriasis, as well (20).

Furthermore, a study conducted in Canada on patients with SLE represented that $3.46 \%$ of lupus patients showed symptoms of psoriasis. The study found that the risk of developing psoriasis among the population of lupus patients was twice that of the general population. Similar results have been obtained regarding gender distribution as psoriasis was more prevalent in the female population. In contrast to our study, the proportion of female to male was higher in patients with lupus who did not show symptoms of Psoriasis (21). Consistent with the Canadian study and in contrast to ours, Tsai et al reported that the risk of developing lupus among psoriatic patients was up to 6-fold more than the general population, with a higher probability among females (22). Perhaps the findings of our study were due to the examination of skin lesions in patients with active lupus or due to the small size of the studied population, males in particular.

In further evaluations, we found no association between the age and duration of SLE among the possessed patients with active SLE who developed psoriasis. Other earlier studies have not reported an association between the age of patients with psoriasis $(10,20)$.

It is noteworthy in our study that no association between lupus activity and psoriasis was detected. Therefore, further studies with a larger population are recommended because the activity of each autoimmune disease may affect the other. Previous studies have found no link between the diagnosis of lupus and Psoriasis, as well. In the study by Tselios et al, the time of lupus onset and the occurrence of psoriasis was not statistically correlated, however the type of plaque-type of psoriasis was remarkably higher than the other types among the population of patients with lupus.

A significant limitation of the current study is the failure to evaluate the correlation between the types of psoriatic lesions with SLE-related. Moreover, another weakness of our study was its cross-sectional design, while a cohort study not only is methodologically strong but also provides a condition to assess the efficacy of therapeutic approaches and effect lupus activity status on the development and control of psoriasis; however, Tselios' and colleagues represented no correlation between the activity of psoriasis and SLE activity status or development. A similar condition was found for SLE, as well (21).

\section{Conclusion}

According to our study, the mean age of SLE patients, the duration of lupus, and the mean score of lupus activity were not associated with psoriasis coexistence, while SLE males were significantly more affected by psoriasis 
concomitantly as compared to females. Further studies are recommended for more definitive results.

\section{Limitations of the study}

The small number of studied population is the most significant limitation of our study. In addition, it is recommended to design a cohort study in order to improve the study power and investigate the coincidence of SLE and psoriasis in course of the disease. Further coincidence rheumatologic studies are recommended.

\section{Authors' contribution}

MK, SHM and AS were the principal investigators of the study. MK, SHM and AS were included in preparing the concept and design. MK, SHM and AS revisited the manuscript and critically evaluated the intellectual contents. All authors participated in preparing the final draft of the manuscript, revised the manuscript and critically evaluated the intellectual contents. All authors have read and approved the content of the manuscript and confirmed the accuracy or integrity of any part of the work.

Conflicts of interest

The authors declare that they have no competing interests.

Ethical considerations

Ethical issues (including plagiarism, data fabrication, double publication) have been completely observed by the authors.

\section{Funding/Support}

This study was sponsored by Isfahan University of Medical Sciences. This study is based on a research project, approved by the Research Vice-Chancellor of Isfahan University of Medical Sciences (Grant\# 396180).

\section{References}

1. Lai N-S, Tsai T-Y, Koo M, Huang K-Y, Tung C-H, Lu M-C. Patterns of ambulatory medical care utilization and rheumatologist consultation predating the diagnosis of systemic lupus erythematosus: a national population-based study. PloS One. 2014;9:e101485. doi: 10.1371/journal.pone.0101485.

2. Agmon-Levin N, Damoiseaux J, Kallenberg C, Sack U, Witte $\mathrm{T}$, Herold $\mathrm{M}$, et al. International recommendations for the assessment of autoantibodies to cellular antigens referred to as anti-nuclear antibodies. Ann Rheum Dis. 2014;73:17-23 . doi: 10.1136/annrheumdis-2013-203863.

3. Eriksson $\mathrm{C}$, Kokkonen $\mathrm{H}$, Johansson $\mathrm{M}$, Hallmans $\mathrm{G}$, Wadell G, Rantapää-Dahlqvist S. Autoantibodies predate the onset of systemic lupus erythematosus in northern Sweden. Arthritis Res. Ther. 2011:13:R30. doi: 10.1186/ar3258

4. Arango M-T, Perricone C, Kivity S, Cipriano E, Ceccarelli F, Valesini $\mathrm{G}$, et al. HLA-DRB1 the notorious gene in the mosaic of autoimmunity. Immunol. Res. 2017;65:82-98. doi: 10.1007/ s12026-016-8817-7.

5. Turner-Stokes T, Lu TY, Ehrenstein MR, Giles I, Rahman A, Isenberg DA. The efficacy of repeated treatment with B-cell depletion therapy in systemic lupus erythematosus: an evaluation. Rheumatology. 2011;50:1401-8. doi: 10.1093/ rheumatology/ker018.
6. Bruyn G. Controversies in lupus: nervous system involvement. Ann Rheum Dis. 1995;54:159-67. doi: 10.1136/ard.54.3.159

7. Moutsopoulos HM, Zampeli E, Vlachoyiannopoulos PG. Systemic Lupus Erythematosus, Antiphospholipid Syndrome, and Mixed Connective Tissue Disease. In: Rheumatology in Questions. Cham: Springer; 2018. p. 59-70. doi: 10.1007/9783-319-71604-6_4.

8. Postal M, Costallat LT, Appenzeller S. Neuropsychiatric manifestations in systemic lupus erythematosus. CNS Drugs. 2011;25:721-36. doi: 10.2165/11591670-000000000-00000.

9. Zhou Q, Mrowietz U, Rostami-Yazdi M. Oxidative stress in the pathogenesis of psoriasis. Free Radical Bio Med. 2009;47:891905. doi: 10.1016/j.freeradbiomed.2009.06.033.

10. Varada S, Gottlieb AB, Merola JF, Saraiya AR, Tintle SJ. Treatment of coexistent psoriasis and lupus erythematosus. J Am Acad Dermatol. 2015;72:253-60. doi: 10.1016/j.jaad.2014.10.038.

11. Ghazizadeh R, Tosa M, Ghazizadeh M. Clinical improvement in psoriasis with treatment of associated hyperlipidemia. Am J Med Sci. 2011;341:394-8. doi: 10.1097/MAJ.0b013e3181ff8eeb

12. Pons-Estel G, Wojdyla D, McGwin Jr G, Magder L, Petri M, Pons-Estel B, et al. The American College of Rheumatology and the Systemic Lupus International Collaborating Clinics classification criteria for systemic lupus erythematosus in two multiethnic cohorts: a commentary. Lupus. 2014;23:3-9. doi: 10.1177/0961203313512883

13. Touma Z, Urowitz M, Gladman D. SLEDAI-2K for a 30-day window. Lupus. 2010;19:49-51. doi: 10.1177/0961203309346505

14. Raychaudhuri SK, Maverakis E, Raychaudhuri SP .Diagnosis and classification of psoriasis. Autoimmun Rev. 2014;13:4905. doi: 10.1016/j.autrev.2014.01.008

15. Kido M, Takeuchi S, Sugiyama N, Esaki H, Nakashima $H$, Yoshida $\mathrm{H}$, et al. T cell-specific overexpression of interleukin-27 receptor a subunit (WSX-1) prevents spontaneous skin inflammation in MRL/lpr mice. Br J Dermatol. 2011;164:121420. doi: 10.1111/j.1365-2133.2011.10244.x.

16. Griffiths CE, Barker JN. Pathogenesis and clinical features of psoriasis. Lancet. 2007;370:263-71. doi: 10.1016/S01406736(07)61128-3.

17. Sticherling M. Psoriasis and autoimmunity. Autoimmun. Rev. 2016;15(12):1167-70. doi: 10.1016/j.autrev.2016.09.004.

18. Plantinga L, Lim SS, Patzer R, McClellan W, Kramer M, Klein $M$, et al. Incidence of end-stage renal disease among newly diagnosed systemic lupus erythematosus patients: the Georgia Lupus Registry. Arthrit Care Res. 2016;68:357-65. doi: 10.1002/acr.22685

19. Berthelot C, Nash J, Duvic M. Coexistent psoriasis and lupus erythematosus treated with alefacept. Am J Clin. Dermatol. 2007;8:47-50. doi: 10.2165/00128071-200708010-00007.

20. Zalla MJ, Muller SA. The coexistence of psoriasis with lupus erythematosus and other photosensitive disorders. Acta Derm Venereol Suppl (Stockh). 1996;195:1.

21. Tselios K, Yap KS-Y, Pakchotanon R, Polachek A, Su J, Urowitz $M B$, et al. Psoriasis in systemic lupus erythematosus: a singlecenter experience. Clin Rheumatol. 2017;36:879-84. doi: 10.1007/s10067-017-3566-0.

22. Tsai T-F, Wang T-S, Hung S-T, Phiona I, Tsai C, Schenkel B, et al. Epidemiology and comorbidities of psoriasis patients in a national database in Taiwan. J Dermatol Sci. 2011;63:40-6. doi: 10.1016/j.jdermsci.2011.03.002 